\title{
Obesidade abdominal no ELSA-Brasil: construção de padrão- ouro latente e avaliação da acurácia de indicadores diagnósticos
}

\author{
Abdominal obesity in ELSA-Brasil (Brazil's Longitudinal Study \\ of Adult Health): construction of a latent gold standard and \\ evaluation of the accuracy of diagnostic indicators
}

\author{
Michaela Eickemberg (https://orcid.org/0000-0002-3625-2221) ${ }^{1}$ \\ Leila Denise Alves Ferreira Amorim (https://orcid.org/0000-0002-1112-2332) ${ }^{1}$ \\ Maria da Conceição Chagas de Almeida (https://orcid.org/0000-0002-4760-4157) ${ }^{2}$ \\ Francisco José Gondim Pitanga (https://orcid.org/0000-0002-1033-8684) ${ }^{1}$ \\ Estela Maria Leão de Aquino (https://orcid.org/0000-0002-8204-1249) ${ }^{1}$ \\ Maria de Jesus Mendes da Fonseca (https://orcid.org/0000-0002-5319-5513) ${ }^{3}$ \\ Sheila Maria Alvim Matos (https://orcid.org/0000-0003-2080-9213) ${ }^{1}$
}

\footnotetext{
${ }^{1}$ Instituto de Saúde Coletiva Universidade Federal da Bahia. R. Basílio da Gama s/no, Canela. 40110-040 Salvador BA Brasil. mieickemberg@ yahoo.com.br ${ }^{2}$ Centro de Pesquisas Gonçalo Moniz, Fiocruz. Salvador BA Brasil. ${ }^{3}$ Escola Nacional de Saúde Pública, Fiocruz. Rio de Janeiro RJ Brasil.
}

\begin{abstract}
This study evaluated the accuracy of abdominal obesity $(A O)$ indicators, defining a latent variable as the gold standard. The study included 12,232 participants of the ELSA-Brasil (Brazil's Longitudinal Study of Adult Health), between 35 and 74 years of age. Three AO indicators were evaluated: waist circumference (WC), waist hip ratio (WHR) and conicity index (C index). Analyses were stratified by sex and race/skin color. All groups had a high prevalence of $A O$, being greater among white men ( $70 \%)$ and black women ( 60\%). A high incidence of WC was observed for men, WHR and C index between men and women for discriminating latent $A O$. The following cutoff points for AO indicators were identified among white, brown and black men, respectively: WC: $89.9 \mathrm{~cm} ; 90.2 \mathrm{~cm}$ and $91.7 \mathrm{~cm}$; WHR: 0.92; 0.92 and 0.90; $C$ index: 1.24; 1.24 and 1.24. The cutoff points identified among white, brown and black women were, respectively: WC: $80.4 \mathrm{~cm}$, $82.7 \mathrm{~cm}$ and $85.4 \mathrm{~cm}$; WHR: 0.82; 0.83 and 0.84; C index: 1.20; 1.22 and 1.19 The WC among men and the WHR and C index among men and women presented high power to discriminate latent $A O$, the $C$ index being the best indicator.
\end{abstract}

Key words Abdominal obesity, Nutritional assessment, Reference standards, Prevalence
Resumo Este estudo avaliou a acurácia de indicadores de obesidade abdominal (OA), definindo uma variável latente como padrão-ouro. Foram estudados 12.232 participantes do ELSA-Brasil de 35 a 74 anos. Avaliou-se três indicadores de OA, estratificados por sexo e raça/cor: circunferência da cintura (CC), razão cintura quadril (RCQ) $e$ indice de conicidade (Índice C). Todos os grupos mostraram elevadas prevalências de OA, maiores entre os homens brancos $(\sim 70 \%)$ e mulheres pretas ( $60 \%)$. Observou-se alta acurácia da CC para homens, RCQ e indice C entre homens e mulheres para discriminar OA latente. Identificou-se os seguintes pontos de corte para os indicadores de OA entre os homens brancos, pardos e pretos, respectivamente: CC: 89,9; 90,2 e 91,7cm; RCQ: 0,92; 0,92 e 0,90; indice C: 1,24; 1,24 e 1,24. Para as mulheres brancas, pardas e pretas, respectivamente, os pontos de corte identificados foram: CC: 80,4; 82,7 e 85,4cm; RCQ: 0,82; 0,83 e 0,84; indice C: 1,$20 ; 1,22$ e 1,19. A CC entre os homens e a $R C Q$ e indice $C$ entre homens e mulheres apresentaram alto poder para discriminar OA latente, sendo o indice $C$ o melhor indicador.

Palavras-chave Obesidade abdominal, Avaliação nutricional, Padrões de referência, Prevalência 


\section{Introdução}

É consensual a associação entre doenças metabólicas e cardiovasculares e obesidade abdominal, e a elevada ocorrência desta no mundo tem sido confirmada em diferentes grupos étnicos por meio de estudos epidemiológicos e clíni$\cos ^{1,2}$. Este particular padrão de obesidade é um desafio para a saúde pública, sendo considerada simultaneamente situação de enfermidade e fator de risco para o desenvolvimento de doenças metabólicas, cardiovasculares e morte, mesmo na ausência de excesso de peso ou obesidade generalizada ${ }^{3}$.

As diferenças na deposição de gordura corporal em homens e mulheres são observadas desde a fase fetal, tornando-se mais pronunciadas com o passar da idade. Além do efeito do dimorfismo sexual, as diferenças étnico-raciais também mostram influência sobre a obesidade abdominal. Sabe-se que essas disparidades provocam desigualdades nos desfechos de saúde relacionados à essa obesidade. Contudo, as evidências são inconclusivas sobre as diferenças no acúmulo de gordura abdominal segundo raça/cor da pele ${ }^{4,5}$.

No Brasil ainda não estão disponíveis dados populacionais acerca da obesidade abdominal estratificados simultaneamente por raça/cor e sexo. Contudo, segundo a Pesquisa Nacional de Saúde, a prevalência desse tipo de obesidade entre mulheres adultas $(21,8 \%)$ é o dobro da ocorrência entre os homens $(52,1 \%)^{6}$.

É recomendado o estabelecimento de pontos de corte segundo raça/cor da pele e sexo específicos em diferentes populações ${ }^{7}$. Particularmente no Brasil, a diversidade étnica, social e cultural pode influenciar o perfil de obesidade abdominal da população por meio de fatores sociais, ambientais, territoriais, comportamentais e políticos. Vários estudos ${ }^{8-11}$ propõem pontos de corte para indicadores de obesidade abdominal, entretanto nenhum ainda forneceu definição para a população brasileira que valorize as diferenças étnico/raciais.

No Brasil, serviços de saúde e pesquisas epidemiológicas utilizam valores de referência para indicadores de obesidade abdominal originalmente desenvolvidos para outras populações ${ }^{7,12}$. Desta forma, podem ser gerados equívocos nas ações de intervenções por meio de erros de classificação nas estimativas de prevalências e, em especial, segundo raça/cor da pele.

No campo da validação de testes diagnósticos, o uso da análise de classes latentes tem sido aplicado quando não se dispõe de informações consideradas padrão-ouro para o diagnóstico da condição sob investigação. Essa abordagem permite combinar resultados de diferentes testes diagnósticos mensuráveis imperfeitos para obter estimativas de prevalência da doença e acurácia. Ao combinar esses indicadores para determinar o diagnóstico de obesidade abdominal latente é possível discriminar indivíduos com o verdadeiro estado de obesidade abdominal, por meio da condição de pertencimento à classe de agrupamento $^{13}$.

Apesar das vantagens no uso dessa técnica, até o presente este é o primeiro estudo que empregou essa metodologia para responder questões específicas à obesidade abdominal. Estudos recentes têm usado a análise de classes latentes para avaliar a acurácia de testes sorológicos no diagnóstico de Leishmaniose visceral ${ }^{14} \mathrm{e}$ de indicadores combinados para o diagnóstico de obesidade generalizada ${ }^{15}$. Esses estudos reforçam a utilização da análise de classes latentes como potencial estratégia analítica para situações em que há ausência de padrões de referência adequados, como ocorre no baseline do ELSA-Brasil.

Os objetivos deste artigo são descrever e empregar um construto de obesidade abdominal como padrão-ouro para apontar a habilidade diagnóstica de três indicadores de obesidade abdominal e estimar as prevalências desta usando os pontos de corte estabelecidos pela literatura e identificados neste estudo.

\section{Metodologia}

\section{Desenho do estudo e população}

O ELSA-Brasil incluiu em sua linha de base 15.105 funcionários públicos, de 35 a 74 anos, vinculados a seis instituições de ensino e pesquisa em diferentes cidades brasileiras (Salvador, Porto Alegre, São Paulo, Rio de Janeiro, Belo Horizonte e Vitória). O estudo foi desenhado para investigar a incidência de doenças cardiovasculares e diabetes e seus determinantes biológicos e sociais. Informações mais detalhadas sobre a metodologia do estudo e o perfil da coorte encontram-se em publicações anteriores ${ }^{16}$. Neste artigo foram usados os dados da linha de base, obtidos entre agosto de 2008 e dezembro de 2010.

Para a realização de entrevistas e exames o estudo contou com equipe treinada e certificada. Procedimentos de garantia da padronização e qualidade na uniformização das condutas adotadas nos seis centros de investigação foram descri- 
Para evitar vieses relacionados à medida de gordura abdominal, excluiu-se os participantes que apresentaram qualquer alteração na distribuição de gordura. Foram excluídos 32 participantes com importantes distrofias corporais e hérnias abdominais. Não ocorreram entre os participantes situações como gestação, realização de cirurgias abdominais recentes, massas intra -abdominais, visceromegalias e ascite.

Para manter uma amostra saudável de participantes, excluiu-se aqueles com triglicerídeos sérico acima de $800 \mathrm{mg} / \mathrm{dL}$ (36 participantes), em uso de medicação hipolipemiante (1974 participantes) e com índice de massa corporal (IMC) acima de $40 \mathrm{Kg} / \mathrm{m}^{2}$ (144 participantes), e participantes que realizaram cirurgia bariátrica anterior ao momento da avaliação (120 participantes). Optou-se por excluir os participantes da raça/cor da pele amarela e indígena devido ao pequeno número de participantes (297 amarelos e 136 indígenas). Excluiu-se os participantes com dados faltantes sobre raça/cor da pele (150 participantes), uma vez que não seria possível definir o construto de obesidade abdominal para indivíduos que não declararam raça/cor da pele. A amostra final foi composta por 12.232 participantes, uma vez que para alguns houve mais de uma condição para exclusão.

\section{Indicadores de obesidade abdominal}

As medidas antropométricas foram aferidas por meio de equipamentos e técnicas padronizadas. Esses procedimentos garantiram padrões internacionais de qualidade e confiabilidade das medidas antropométricas no ELSA-Brasil ${ }^{17}$.

A circunferência da cintura (CC) foi medida no ponto médio entre a borda inferior do arco costal e a crista ilíaca, na linha axilar média e classificada segundo a International Diabetes Federation (IDF) ( $\geq 80 \mathrm{~cm}$ para mulheres $\mathrm{e} \geq 90 \mathrm{~cm}$ para homens $)^{7}$. A circunferência do quadril foi aferida na protrusão máxima dos músculos glúteos, sobre a vestimenta. Essas medidas foram realizadas com auxílio de fita métrica inelástica e flexível ${ }^{18,19}$.

As circunferências da cintura e do quadril foram usadas para determinar o tipo de distribuição de gordura corporal, segundo a razão cintura-quadril (RCQ) (androide: $\geq 0,85$ para mulheres e $\geq 0,90$ para homens) $)^{12}$. O índice de conicidade (índice C) foi calculado por meio da fórmula: Índice $\mathrm{C}=\mathrm{CC} / 0,109 \times \sqrt{ }$ Peso/Altura ${ }^{20} \mathrm{e}$ classificado de acordo com Pitanga e Lessa ${ }^{11}$ (> 1,18 para mulheres e $>1,25$ para homens).

\section{Construto de obesidade abdominal}

Como não houve, neste estudo, um indicador padrão-ouro perfeito para o diagnóstico de obesidade abdominal, então foi definida uma variável latente. Para a definição desse construto utilizou-se os indicadores CC, RCQ e índice $\mathrm{C}$ em suas naturezas categóricas. $\mathrm{O}$ construto obesidade abdominal foi considerado como padrão-ouro para descrever as categorias presença ou ausência de obesidade abdominal.

\section{Outras variáveis}

As demais variáveis foram selecionadas pela estreita relação com obesidade abdominal evidenciadas na literatura ${ }^{21}$. Idade foi categorizada em 35 a 49 anos; 50 a 59 anos e mais de 60 anos. Nível educacional foi estratificado em ensino fundamental incompleto e completo; ensino médio completo e ensino superior completo. A situação conjugal foi classificada em solteiro, separado ou viúvo e casado ou em união estável. Raça/cor da pele foi autorrelatada e categorizada em branca, parda e preta. A ingestão de bebidas alcoólicas foi classificada em nunca fez uso, ex-usuário, usuário atual e usuário em quantidade excessiva. Foi definida quantidade excessiva para homens $\geq$ $210 \mathrm{~g}$ álcool por semana; e para mulheres $\geq 140 \mathrm{~g}$ álcool por semana ${ }^{22}$. Para o tabagismo, os participantes foram estratificados em não fumantes, ex-fumantes e fumantes atuais. A atividade física foi avaliada por meio do International Physical Activity Questionnary (IPAQ), sendo aplicados os domínios lazer da versão longa ${ }^{23}$, e classificada, considerando minutos e dias da semana, em forte, moderada e fraca ${ }^{24}$.

$O$ peso corporal e a altura foram aferidos com os participantes usando a vestimenta do estudo, sem sapatos e adereços. Utilizou-se para aferição balanças da marca Toledo ${ }^{\circledR}$ e estadiômetro $\mathrm{Seca}^{\circledR}$, com protocolos padronizados ${ }^{25}$. Essas medidas foram empregadas no cálculo do IMC (peso/altura ${ }^{2}$ ), usado em natureza contínua. 


\section{Análise estatística}

Estatística descritiva foi usada para avaliar a distribuição dos participantes segundo as características de interesse. Variáveis contínuas com distribuição simétrica foram apresentadas por meio de média e desvio padrão. Todas as análises foram estratificadas por sexo e raça/cor da pele. Foi adotado nível de significância de 5\% e utilizados os softwares Stata versão 12.1 (Stata Corporation, College Station, Texas, USA) e Mplus versão 5 (Muthén \& Muthén, Los Angeles, USA).

A seguir serão descritas as etapas realizadas na análise estatística.

\section{Passo 1 - Sorteio do banco de dados}

Para atender aos objetivos propostos e definir a obesidade abdominal latente por sexo e raça/ cor da pele, o banco de dados foi separado em seis subconjuntos de dados para representar cada um desses estratos. Em seguida, obteve-se para cada um dos estratos dois subconjuntos aleatórios para as análises subsequentes.

Portanto, para a definição da obesidade abdominal latente, um subconjunto aleatório com $50 \%$ de participantes de cada um dos seis bancos ( $\mathrm{n}=6114)$ foi usado como treinamento/preparação para estimar o modelo de classes latentes. Com estes dados foram descritos os padrões de resposta de cada classe latente, estimando-se sua prevalência e relação com os indicadores de obesidade abdominal. No outro subconjunto aleatório com características similares, com os restantes $50 \%$ de cada um dos seis bancos $(n=6118)$, aplicou-se os padrões de resposta da variável latente obesidade abdominal da primeira etapa em uma validação cruzada para que fosse possível encontrar os pontos de corte para os indicadores de obesidade abdominal ${ }^{26}$.

\section{Passo 2 - Análise de Classes Latentes}

A análise de classes latentes é indicada quando há um conjunto de variáveis categóricas condicionalmente independentes, possibilitando a representação de dados complexos de forma mais compreensível, organizando indivíduos em dois ou mais subgrupos homogêneos. A interpretação do modelo de classes latentes é baseada no cálculo da probabilidade do indivíduo fazer parte de determinada categoria de uma variável, pertencendo a determinado grupo, com características que os difere dos demais ${ }^{26}$.

Esta análise foi aplicada em 50\% dos dados, para identificar classes de indivíduos mutuamente exclusivas acerca da obesidade abdominal (presença ou ausência), ou subgrupos com padrões similares de respostas para um conjunto de indicadores de obesidade abdominal. Foram considerados os indicadores CC, RCQ e índice C, em natureza categórica, segundo os critérios da literatura anteriormente mencionados. A análise de classes latentes produziu um padrão de respostas combinadas que foi aplicado no segundo subconjunto aleatório de dados, possibilitando a classificação de obesidade abdominal latente.

Os modelos de classes latentes foram avaliados com base na entropia e no critério de bondade de ajuste do modelo [critério de informação Bayesiano (BIC), critério de informação Akaike (AIC) e critério de informação Bayesiano ajustado pelo tamanho da amostra (BIC ajustado) $]^{26}$. Foram estimadas probabilidades condicionais e prevalências de obesidade abdominal. Também foram definidos os padrões de respostas dos indicadores para o diagnóstico de obesidade abdominal.

\section{Passo 3 - Análise com Receiver Operating Characteristic Curves (Curva ROC)}

Considerando-se os resultados da análise de classes latentes obtidos com base na primeira subamostra aleatória de 6.114 participantes, aplicou-se o padrão de respostas da variável latente obesidade abdominal na segunda subamostra (6.118 participantes). Essa nova variável foi adotada como padrão-ouro na análise com Curvas ROC para, então, avaliar a habilidade diagnóstica dos indicadores de obesidade abdominal isolados. Estimou-se a sensibilidade, a especificidade, a área sob a curva (acurácia), os pontos de corte para esses indicadores e o índice de Youden.

$\mathrm{O}$ índice de Youden ([maximum (sensitivity + specificity -1$)]$ ) fornece um critério para a escolha do melhor ponto de corte ao indicar a máxima taxa global de correta classificação que um marcador diagnóstico pode alcançar, atuando como uma medida direta de acurácia diagnóstica do ponto de corte. Os valores do índice de Youden variam de 0 a 1 , sendo 1 a acurácia perfeita, ou seja, a completa separação da distribuição dos valores dos marcadores entre doentes e não doentes e 0 a completa sobreposição, ou a incapacidade do marcador diagnóstico discriminar doentes e não doentes ${ }^{27}$.

\section{Passo 4 - Prevalência de obesidade abdominal}

A partir dos pontos de corte identificados na análise de Curva ROC para os indicadores de obesidade abdominal, as prevalências de obe- 
sidade abdominal e os respectivos intervalos de 95\% de confiança foram estimados. Para comparação foram calculadas, nos mesmos grupos, as prevalências de obesidade abdominal com base nos pontos de corte propostos pela literatura e na análise de classes latentes.

\section{Considerações éticas}

O ELSA-Brasil foi aprovado pelos Comitês de Ética em Pesquisa de cada instituição envolvida e atende a todos os requisitos éticos necessários a uma pesquisa científica realizada com seres humanos.

\section{Resultados}

Homens e mulheres de raça/cor preta apresentaram menores proporções de ensino superior completo comparado aos brancos e pardos. Com relação à situação conjugal, observou-se menor proporção de casadas/união estável entre as mulheres pretas. A atividade física classificada como fraca foi mais frequente entre homens e mulheres negros (pardos e pretos) em comparação aos brancos. Os valores médios de IMC foram maiores entre mulheres pretas (Tabela 1).

Na primeira subamostra aleatória cada participante foi classificado na classe latente associada à maior probabilidade de pertencimento. Para todos os grupos analisados, a maioria dos participantes foi classificada com obesidade abdominal, sendo esta mais expressiva entre homens bran$\cos (69,9 \%)$ e mulheres pardas e pretas $(63,3 \%$ e $60,0 \%$, respectivamente). Observou-se maior prevalência de obesidade abdominal entre os homens (Tabela 2).

Para ambos os sexos, em todas as categorias de raça/cor da pele, as frequências na classe latente que indica presença de obesidade abdominal variaram entre $77 \%$ e $99,1 \%$ para os três indicadores (CC, RCQ e Índice C). Entre os homens, a RCQ foi o indicador do componente latente com as maiores frequências, seguido do Índice C e CC. Para as mulheres, as maiores frequências de obesidade abdominal foram observadas para a CC, seguidas pelo Índice C e RCQ (Tabela 2).

Para avaliar a bondade do ajuste dos modelos da análise de classes latentes e a capacidade de discriminar indivíduos nas diferentes classes todos os modelos foram criteriosamente analisados. Ao comparar os modelos com duas e três classes, os valores dos critérios de informação indicaram como mais apropriado o modelo com duas classes latentes (entropia entre 0,865 e 0,917 nos homens e entre 0,881 e 0,867 nas mulheres dados não mostrados).

O padrão de respostas obtido na análise de classes latentes mostrou que participantes com ao menos dois dos três indicadores diagnósticos de obesidade abdominal positivos foram classificados com presença de obesidade abdominal. $\mathrm{O}$ padrão de resposta mais frequente foi observado para o diagnóstico positivo de obesidade abdominal para os três indicadores (CC, RCQ e índice C) de $37,4 \%$ a $57,5 \%$ em todos os subgrupos avaliados (Tabela 3).

Aplicou-se o padrão de resposta da análise de classes latentes nos outros $50 \%$ da subamostra aleatória e, então, foi utilizada a curva ROC para investigar separadamente cada um dos indicadores de obesidade abdominal usando a obesidade abdominal latente como padrão-ouro. As maiores áreas sob a curva ROC foram obtidas para o índice $\mathrm{C}$ em todos os grupos (Tabela 4). Também se observou alta acurácia da CC para homens, e da RCQ para homens e mulheres em distinguir participantes com e sem obesidade abdominal em todas as categorias de raça/cor da pele.

Para a CC, os pontos de corte mais apropriados para discriminar obesidade abdominal latente estão descritos na Tabela 4, sendo mais elevado para homens pretos $(91,7 \mathrm{~cm})$. A RCQ mostrou os maiores pontos de corte para homens brancos e pardos $(0,92)$ e mulheres pretas $(0,84)$.

Para efeito de comparação com as prevalências de obesidade abdominal geradas a partir dos pontos de corte identificados, a Figura 1 fornece as prevalências estimadas a partir dos pontos de corte da literatura ${ }^{7,11,12}$ para os três indicadores. Todos os grupos apresentaram elevadas prevalências de obesidade abdominal. Para o sexo masculino, os maiores valores foram observados pelo indicador RCQ. Entre as mulheres, as maiores prevalências foram encontradas usando CC.

As prevalências de obesidade abdominal estimadas a partir dos pontos de corte identificados na Curva ROC são apresentadas na Figura 2. As maiores prevalências de obesidade abdominal mantiveram-se entre os homens brancos (70\%) e pardos $(67,4 \%)$ e mulheres pardas $(59,5)$ e pretas (62\%). Os indicadores com maiores prevalências foram o índice $\mathrm{C}$ entre os homens brancos e pardos e a RCQ entre os brancos e pretos. No grupo das mulheres, o indicador com a maior prevalência de obesidade abdominal foi o índice $\mathrm{C}$ entre as pretas. 
Tabela 1. Caracterização dos participantes da linha de base do ELSA-Brasil, segundo sexo e raça/cor da pele. ELSA-Brasil, 2008-2010.

\begin{tabular}{|c|c|c|c|c|c|c|}
\hline & \multicolumn{3}{|c|}{ Homens } & \multicolumn{3}{|c|}{ Mulheres } \\
\hline & $\begin{array}{l}\text { Brancos } \\
\mathrm{N}=2945\end{array}$ & $\begin{array}{c}\text { Pardos } \\
\mathrm{N}=1813\end{array}$ & $\begin{array}{c}\text { Pretos } \\
\mathrm{N}=841\end{array}$ & $\begin{array}{l}\text { Brancas } \\
\mathrm{N}=3501\end{array}$ & $\begin{array}{c}\text { Pardas } \\
\mathrm{N}=1888\end{array}$ & $\begin{array}{c}\text { Pretas } \\
\mathrm{N}=1244\end{array}$ \\
\hline \multicolumn{7}{|l|}{ Idade, n (\%) } \\
\hline $35-49$ anos & $1343(45,6)$ & $921(50,8)$ & $386(45,9)$ & $1642(46,9)$ & $895(47,4)$ & $588(47,2)$ \\
\hline $50-59$ anos & $1006(34,1)$ & $637(35,1)$ & $307(36,5)$ & $1229(35,1)$ & $690(36,5)$ & $455(36,5)$ \\
\hline$\geq 60$ anos & $596(20,2)$ & $255(14,1)$ & $148(17,6)$ & $630(17,9)$ & $303(16,0)$ & $201(16,1)$ \\
\hline \multicolumn{7}{|l|}{ Nível educacional, n (\%) } \\
\hline $\begin{array}{l}\text { Ensino fundamental } \\
\text { incompleto + completo }\end{array}$ & $287(9,7)$ & $378(20,8)$ & $247(29,3)$ & $170(4,8)$ & $239(12,6)$ & $199(16,0)$ \\
\hline Ensino médio completo & $782(26,5)$ & $783(43,1)$ & $404(48,1)$ & $959(27,3)$ & $794(42,1)$ & $655(52,6)$ \\
\hline Superior completo & $1876(63,7)$ & $652(35,9)$ & $190(22,5)$ & $2372(67,7)$ & $855(45,2)$ & $390(31,3)$ \\
\hline \multicolumn{7}{|l|}{ Situação conjugal, n (\%) } \\
\hline Solteiro & $167(5,6)$ & $99(5,4)$ & $39(4,6)$ & $448(12,8)$ & $218(11,5)$ & $220(17,6)$ \\
\hline Separado/viúvo & $385(13,1)$ & $234(12,9)$ & $118(14,0)$ & $1063(30,3)$ & $662(35,1)$ & $434(34,8)$ \\
\hline Casado/união estável & $2393(81,2)$ & $1480(81,2)$ & $684(81,3)$ & $1990(56,8)$ & $1008(53,3)$ & $590(47,4)$ \\
\hline \multicolumn{7}{|l|}{ Uso de álcool, n(\%) } \\
\hline Nunca fez uso & $102(3,4)$ & $99(5,4)$ & $45(5,3)$ & $412(11,7)$ & $361(19,1)$ & $247(19,8)$ \\
\hline Ex usuário & $494(16,7)$ & $408(22,5)$ & $195(23,1)$ & $576(16,4)$ & $463(24,5)$ & $275(22,1)$ \\
\hline Usuário atual & $2025(68,7)$ & $1065(58,7)$ & $477(56,7)$ & $2381(68,1)$ & $1000(52,9)$ & $667(53,7)$ \\
\hline $\begin{array}{l}\text { Usuário de quantidade } \\
\text { excessiva }\end{array}$ & $324(11,1)$ & $241(13,2)$ & $124(14,7)$ & $127(3,6)$ & $63(3,3)$ & $53(4,2)$ \\
\hline \multicolumn{7}{|l|}{ Tabagismo, $\mathrm{n}(\%)$} \\
\hline Nunca fumou & $1542(52,3)$ & $939(51,8)$ & $426(50,6)$ & $2152(61,4)$ & $1229(65,1)$ & $804(64,6)$ \\
\hline Ex fumante & $1003(34,1)$ & $573(31,6)$ & $275(32,7)$ & $914(26,1)$ & $433(22,9)$ & $267(21,4)$ \\
\hline Fumante atual & $400(13,5)$ & $300(16,5)$ & $140(16,6)$ & $435(12,4)$ & $226(11,9)$ & $173(13,9)$ \\
\hline \multicolumn{7}{|l|}{ Atividade física no lazer, $\mathrm{n}(\%)$} \\
\hline Forte & $292(10,0)$ & $167(9,3)$ & $65(7,7)$ & $226(6,5)$ & $83(4,4)$ & $43(3,4)$ \\
\hline Moderada & $540(18,6)$ & $263(14,9)$ & $110(13,1)$ & $554(16,1)$ & $231(12,4)$ & $137(11,1)$ \\
\hline Fraca & $2068(71,3)$ & $1357(75,7)$ & $659(79,1)$ & $2655(77,2)$ & $1548(83,1)$ & $1053(85,4)$ \\
\hline IMC, $(\mathrm{Kg} / \mathrm{m} 2)$ média (DP) & $26,69(4,0)$ & $26,48(4,0)$ & $27,01(3,9)$ & $26,07(4,5)$ & $26,82(4,5)$ & $27,92(4,9)$ \\
\hline CC, $(\mathrm{cm})$ média $(\mathrm{DP})$ & $95,1(11,4)$ & $93,5(11,1)$ & $93,9(10,8)$ & $85,4(11,6)$ & $87,3(11,7)$ & $89,2(12,0)$ \\
\hline $\mathrm{CQ},(\mathrm{cm})$ média $(\mathrm{DP})$ & $100,0(7,5)$ & $99,1(8,0)$ & $100,1(7,4)$ & $101,9(9,7)$ & $102,6(9,3)$ & $104,2(9,9)$ \\
\hline RCQ, média (DP) & $0,94(0,0)$ & $0,94(0,0)$ & $0,93(0,0)$ & $0,83(0,0)$ & $0,85(0,0)$ & $0,85(0,0)$ \\
\hline Índice C, média (DP) & $1,28(0,0)$ & $1,27(0,0)$ & $1,26(0,0)$ & $1,21(0,0)$ & $1,22(0,0)$ & $1,22(0,0)$ \\
\hline
\end{tabular}

\section{Discussão}

No presente estudo, a ausência de uma medida reconhecidamente de referência para determinar o diagnóstico de obesidade abdominal deu lugar à avaliação conjunta de indicadores bem explorados na literatura para determinar a obesidade abdominal por meio de análise de classes latentes. Estudos prévios mostram que indicadores imperfeitos combinados fornecem informações complementares, podendo reduzir erros de classificação do diagnóstico ao aproximarem-se da verdadeira condição sob investigação ${ }^{13,15}$.

A CC, a RCQ e o índice C mostraram excelente acurácia para discriminar obesidade abdominal latente, tendo o índice $\mathrm{C}$ as mais elevadas áreas sob a Curva ROC (acima de 90\%). Estudos realizados no nordeste do Brasil, com predominância da população negra, em amostras comunitárias, ambulatoriais e com funcionárias 
Tabela 2. Estimativas usando análise de classes latentes para o construto obesidade abdominal, de acordo com sexo e raça/cor da pele. ELSA- Brasil, 2008-2010.

\begin{tabular}{|c|c|c|c|c|c|c|c|c|c|}
\hline \multirow{2}{*}{$\begin{array}{c}\text { Obesidade } \\
\text { abdominal }\end{array}$} & \multicolumn{3}{|c|}{ Brancos } & \multicolumn{3}{|c|}{ Pardos } & \multicolumn{3}{|c|}{ Pretos } \\
\hline & Geral & $+(\%)$ & $-(\%)$ & Geral & $+(\%)$ & $-(\%)$ & Geral & $+(\%)$ & $-(\%)$ \\
\hline Homens & \multicolumn{3}{|c|}{$n=1472$} & \multicolumn{3}{|c|}{$n=906$} & \multicolumn{3}{|c|}{$n=420$} \\
\hline $\begin{array}{l}\mathrm{N}^{\circ} \text { de indivíduos } \\
\text { nas classes (\%) }\end{array}$ & - & $\begin{array}{r}1029 \\
(69,9)\end{array}$ & $\begin{array}{r}443 \\
(30,1)\end{array}$ & - & $\begin{array}{r}598 \\
(66,0)\end{array}$ & $\begin{array}{r}308 \\
(34,0)\end{array}$ & - & $\begin{array}{r}279 \\
(66,4)\end{array}$ & $\begin{array}{r}141 \\
(33,6)\end{array}$ \\
\hline $\mathrm{CC}$ & 65,6 & 90,3 & 9,4 & 60,2 & 86,4 & 9,1 & 64,8 & 87,1 & 22,5 \\
\hline RCQ & 75,7 & 99,1 & 22,3 & 72,4 & 97,6 & 23,4 & 71,9 & 97,5 & 76,6 \\
\hline Índice C & 65,6 & 92,5 & 4,5 & 61,8 & 92,6 & 1,9 & 60,0 & 90,3 & 2,6 \\
\hline Mulheres & \multicolumn{3}{|c|}{$n=1750$} & \multicolumn{3}{|c|}{$\mathrm{n}=944$} & \multicolumn{3}{|c|}{$n=622$} \\
\hline $\begin{array}{l}\mathrm{N}^{\circ} \text { de indivíduos } \\
\text { nas classes (\%) }\end{array}$ & - & $\begin{array}{r}922 \\
(52,7)\end{array}$ & $\begin{array}{r}828 \\
(47,3)\end{array}$ & - & $\begin{array}{r}598 \\
(63,3)\end{array}$ & $\begin{array}{r}346 \\
(36,7)\end{array}$ & - & $\begin{array}{r}373 \\
(60,0)\end{array}$ & $\begin{array}{r}249 \\
(40,0)\end{array}$ \\
\hline $\mathrm{CC}$ & 64,4 & 97,5 & 28,4 & 74,4 & 97,0 & 37,3 & 75,6 & 97,0 & 44,7 \\
\hline RCQ & 41,2 & 77,0 & 2,2 & 49,9 & 78,1 & 3,8 & 51,4 & 84,4 & 4,1 \\
\hline Índice C & 53,7 & 95,6 & 8,0 & 64,0 & 95,4 & 12,7 & 58,0 & 94,1 & 6,2 \\
\hline
\end{tabular}

Classificação de indivíduos usando o método de pertencimento mais provável (most likely) às classes latentes. CC: circunferência da cintura; RCQ: razão cintura quadril; Índice C: índice de conicidade; +: presença de obesidade abdominal, -: ausência de obesidade abdominal pelo modelo de análise de classes latentes.

Tabela 3. Padrões de resposta dos indicadores de obesidade abdominal e suas respectivas frequências, segundo sexo e cor ou raça, e atribuição da classe latente para diagnóstico de obesidade abdominal, baseados no modelo de análise de classes latentes. ELSA-Brasil, 2008-2010.

\begin{tabular}{|c|c|c|c|c|c|c|c|c|c|}
\hline \multirow{3}{*}{$\mathrm{CC}$} & \multirow{3}{*}{ RCQ } & \multirow{3}{*}{$\begin{array}{c}\text { Índice } \\
\mathrm{C}\end{array}$} & \multicolumn{3}{|c|}{ Homens } & \multicolumn{3}{|c|}{ Mulheres } & \multirow{3}{*}{$\begin{array}{c}\text { Obesidade } \\
\text { abdomina } \\
\text { baseada na } \\
\text { ACL }\end{array}$} \\
\hline & & & $\begin{array}{c}\text { Brancos } \\
1472\end{array}$ & $\begin{array}{c}\text { Pardos } \\
906\end{array}$ & $\begin{array}{c}\text { Pretos } \\
420\end{array}$ & $\begin{array}{c}\text { Brancas } \\
1750\end{array}$ & $\begin{array}{c}\text { Pardas } \\
944\end{array}$ & $\begin{array}{c}\text { Pretas } \\
622\end{array}$ & \\
\hline & & & $\%$ & $\%$ & $\%$ & $\%$ & $\%$ & $\%$ & \\
\hline 1 & 1 & 1 & 57,5 & 51,5 & 50,2 & 37,4 & 44,9 & 45,4 & + \\
\hline 1 & 1 & 0 & 5,2 & 4,8 & 7,1 & 2,0 & 2,6 & 3,5 & + \\
\hline 1 & 0 & 1 & 0,6 & 1,3 & 1,4 & 12,2 & 14,3 & 9,4 & + \\
\hline 0 & 1 & 1 & 6,4 & 8,2 & 7,6 & 1,0 & 1,4 & 1,4 & + \\
\hline 0 & 0 & 0 & 20,5 & 23,1 & 20,0 & 30,8 & 20,0 & 20,4 & - \\
\hline 0 & 0 & 1 & 1,0 & 0,6 & 0,7 & 2,9 & 3,2 & 1,6 & - \\
\hline 0 & 1 & 0 & 6,3 & 7,7 & 6,9 & 0,7 & 0,8 & 0,9 & - \\
\hline 1 & 0 & 0 & 2,1 & 2,4 & 5,9 & 12,7 & 12,5 & 17,0 & - \\
\hline
\end{tabular}

$\mathrm{N}=$ 6114. CC: circunferência da cintura; RCQ: razão cintura quadril; Índice C: índice de conicidade; ACL: análise de classes latentes. Atribuição da classe latente: +: presença de obesidade abdominal; -: ausência de obesidade abdominal.

de universidade pública, também encontraram o índice $\mathrm{C}$ com o melhor poder discriminatório dentre outros indicadores ${ }^{11,28}$.

Pontos de corte semelhantes aos encontrados neste estudo para o índice C entre os homens foram propostos no Brasil e nos EUA (1,25 e 1,26 no Brasil sem estratificação da raça/cor da pele; $1,23$ entre homens americanos brancos) $)^{28-30}$. Valores dos pontos de corte mais baixos foram observados entre homens residentes no sul do Brasil $(1,18)^{10}$ e homens norte-americanos pre- tos $(1,19)^{30}$. Para as mulheres foram observados pontos de corte divergentes entre estudos brasileiros $^{28,29}$, variando de 1,18 a 1,25. Mulheres do sul do Brasil $(1,15)^{10}$, norte americanas brancas e pretas $(1,13 \text { e } 1,17)^{30}$ e chinesas $(1,12)^{31}$ apresentaram valores menores.

A classificação dos valores obtidos por meio do Índice C, desenvolvido por Valdez et al. ${ }^{20}$, reflete a distribuição de gordura corporal que parte do formato em cilindro (sem acúmulo excessivo de gordura) para um duplo cone com base co- 
Tabela 4. Pontos de corte e medidas de desempenho diagnóstico para os indicadores de obesidade abdominal por sexo e raça/cor da pele, usando padrão ouro latente. ELSA-Brasil, 2008-2010.

\begin{tabular}{|c|c|c|c|c|c|c|}
\hline & \multicolumn{3}{|c|}{ Homens } & \multicolumn{3}{|c|}{ Mulheres } \\
\hline & $\begin{array}{c}\text { Brancos } \\
\mathrm{N}=1473\end{array}$ & $\begin{array}{c}\text { Pardos } \\
\mathrm{N}=907\end{array}$ & $\begin{array}{c}\text { Pretos } \\
\mathrm{N}=421\end{array}$ & $\begin{array}{c}\text { Brancas } \\
\mathrm{N}=1751\end{array}$ & $\begin{array}{c}\text { Pardas } \\
\mathrm{N}=944\end{array}$ & $\begin{array}{c}\text { Pretas } \\
\mathrm{N}=622\end{array}$ \\
\hline \multicolumn{7}{|l|}{$\mathrm{CC}$} \\
\hline Ponto de corte $(\mathrm{cm})$ & 89,9 & 90,2 & 91,7 & 80,4 & 82,7 & 85,4 \\
\hline Sensibilidade (\%) & 91 & 85 & 85 & 96 & 90 & 91 \\
\hline Especificidade (\%) & 91 & 92 & 94 & 77 & 80 & 81 \\
\hline Área sob a curva & 0,91 & 0,89 & 0,90 & 0,87 & 0,85 & 0,86 \\
\hline Índice de Youden & 0,82 & 0,77 & 0,79 & 0,73 & 0,69 & 0,72 \\
\hline \multicolumn{7}{|l|}{ RCQ } \\
\hline Ponto de corte & 0,92 & 0,92 & 0,90 & 0,82 & 0,83 & 0,84 \\
\hline Sensibilidade (\%) & 88 & 90 & 96 & 89 & 84 & 87 \\
\hline Especificidade (\%) & 93 & 94 & 88 & 89 & 96 & 95 \\
\hline Área sob a curva & 0,90 & 0,92 & 0,92 & 0,89 & 0,90 & 0,91 \\
\hline Índice de Youden & 0,81 & 0,83 & 0,83 & 0,78 & 0,79 & 0,81 \\
\hline \multicolumn{7}{|l|}{ Índice C } \\
\hline Ponto de corte & 1,24 & 1,24 & 1,24 & 1,20 & 1,22 & 1,19 \\
\hline Sensibilidade (\%) & 94 & 92 & 88 & 89 & 83 & 95 \\
\hline Especificidade (\%) & 97 & 99 & 98 & 91 & 97 & 88 \\
\hline Área sob a curva & 0,96 & 0,96 & 0,93 & 0,90 & 0,90 & 0,92 \\
\hline Índice de Youden & 0,91 & 0,91 & 0,85 & 0,80 & 0,80 & 0,83 \\
\hline
\end{tabular}

CC: circunferência da cintura; RCQ: razão cintura quadril; Índice C: índice de conicidade.

mum (com excessivo acúmulo de gordura abdominal). Segundo a equação matemática que dá origem ao Índice $\mathrm{C}$, o denominador determina a forma corporal influenciada pelo peso e altura individuais. Neste estudo, participantes de raça/ cor da pele autodeclarada preta apresentaram os maiores valores de IMC, em ambos os sexos. Possivelmente essa característica influenciou o perfil dos achados acerca do Índice $\mathrm{C}$ e seus respectivos pontos de corte maiores que dos estudos realizados com população predominantemente branca.

$\mathrm{O}$ índice $\mathrm{C}$ parece ser um bom discriminador de obesidade abdominal em situações nas quais há considerável parcela de população negra (pretos e pardos), mas ainda é pouco estudado ${ }^{29}$. A maior dificuldade em sua utilização rotineira é a falta de pontos de corte consensuais para referência e o cálculo do denominador em sua fórmula. Para facilitar o uso deste indicador nos serviços de saúde e em pesquisas, Pitanga e Lessa ${ }^{29}$ elaboraram uma planilha com valores prefixados. A vantagem do índice $\mathrm{C}$ sobre os demais indicadores é considerar peso e altura no cálculo, permitindo a comparação entre grupos com diferentes valores dessas variáveis.

Os pontos de corte da CC definidos neste estudo para homens e mulheres foram mais elevados entre participantes de raça/cor da pele preta. Alguns estudos internacionais encontraram maior quantidade de gordura em grupos de raça/cor da pele preta em comparação à branca ${ }^{4,32}$. Apesar da dificuldade em encontrar estudos sobre pontos de corte entre etnias, os valores aqui descritos foram menores que as recomendações sugeridas pela OMS (mulheres $88 \mathrm{~cm}$ e homens $102 \mathrm{~cm})^{12}$. Contudo, para mulheres brancas e homens os valores observados aproximaram-se aos recomendados pela IDF - mulheres $80 \mathrm{~cm}$ e homens $90 \mathrm{~cm}^{7}$. Resultados semelhantes aos deste estudo para participantes pardos e pretos foram encontrados em pesquisas realizadas no nordeste brasileiro: para mulheres $83 \mathrm{~cm}^{11} ; 84 \mathrm{~cm}^{9}$ e para homens $92,2 \mathrm{~cm}^{33}$. Do mesmo modo, resultados para homens brancos foram similares aos encontrados em homens brasileiros da região sul (homens $89,5 \mathrm{~cm}$ ) e também para mulheres pretas $(86,2 \mathrm{~cm})^{10}$.

Um estudo realizado com adultos de 30 a 74 anos de Salvador mostrou resultados concordantes para a RCQ (0,83 para mulheres e 0,92 para homens $)^{11}$. As recomendações da OMS são próximas aos pontos de corte atuais para homens e mulheres de raça/cor da pele autodeclarada preta $(0,90 \text { e } 0,85 \text { respectivamente })^{12}$. Embora a RCQ determine a distribuição de gordura corporal, 

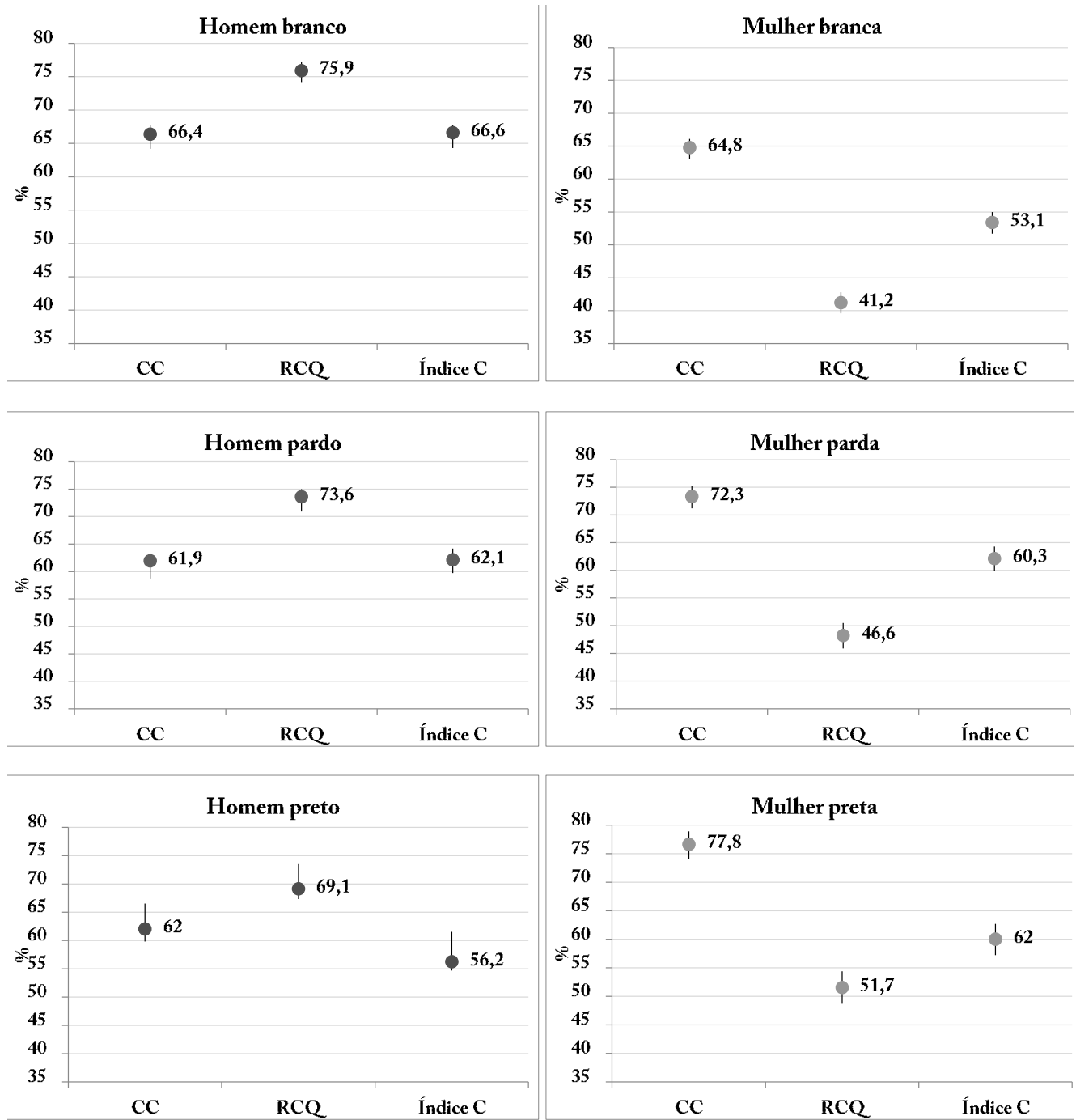

Figura 1. Prevalências de obesidade abdominal e respectivos intervalos de $95 \%$ de confiança para os indicadores de obesidade abdominal, de acordo com sexo e raça/cor da pele segundo os pontos de corte propostos pela literatura. ELSA-Brasil 2008-2010.

CC: circunferência da cintura; RCQ: razão cintura quadril; Índice C: índice de conicidade.

esta é afetada pela variação conjunta dessas medidas CC e circunferência do quadril. Além disso, esse indicador não considera a estrutura óssea do quadril, que pode variar entre populações ${ }^{33}$. Assim, a RCQ pode se manter constante ainda que os sujeitos tenham diferentes quantidades de gordura abdominal e gluteofemural, como observado no atual estudo para homens brancos e pardos e mulheres pardas e pretas com diferentes valores de CC e circunferência do quadril.
As maiores prevalências gerais de obesidade abdominal foram observadas entre os homens brancos $(69,9 \%)$ e as mulheres pardas $(63,3 \%)$ e, como esperado, maior entre os homens (análise de classes latentes). Esse perfil se manteve ao estimar a prevalência entre os indicadores por meio dos pontos de corte definidos na atual pesquisa. Resultados similares foram observados nos EUA com dados do National Health and Nutrition Examination Survey (NHANES) ${ }^{2}$, com maiores 


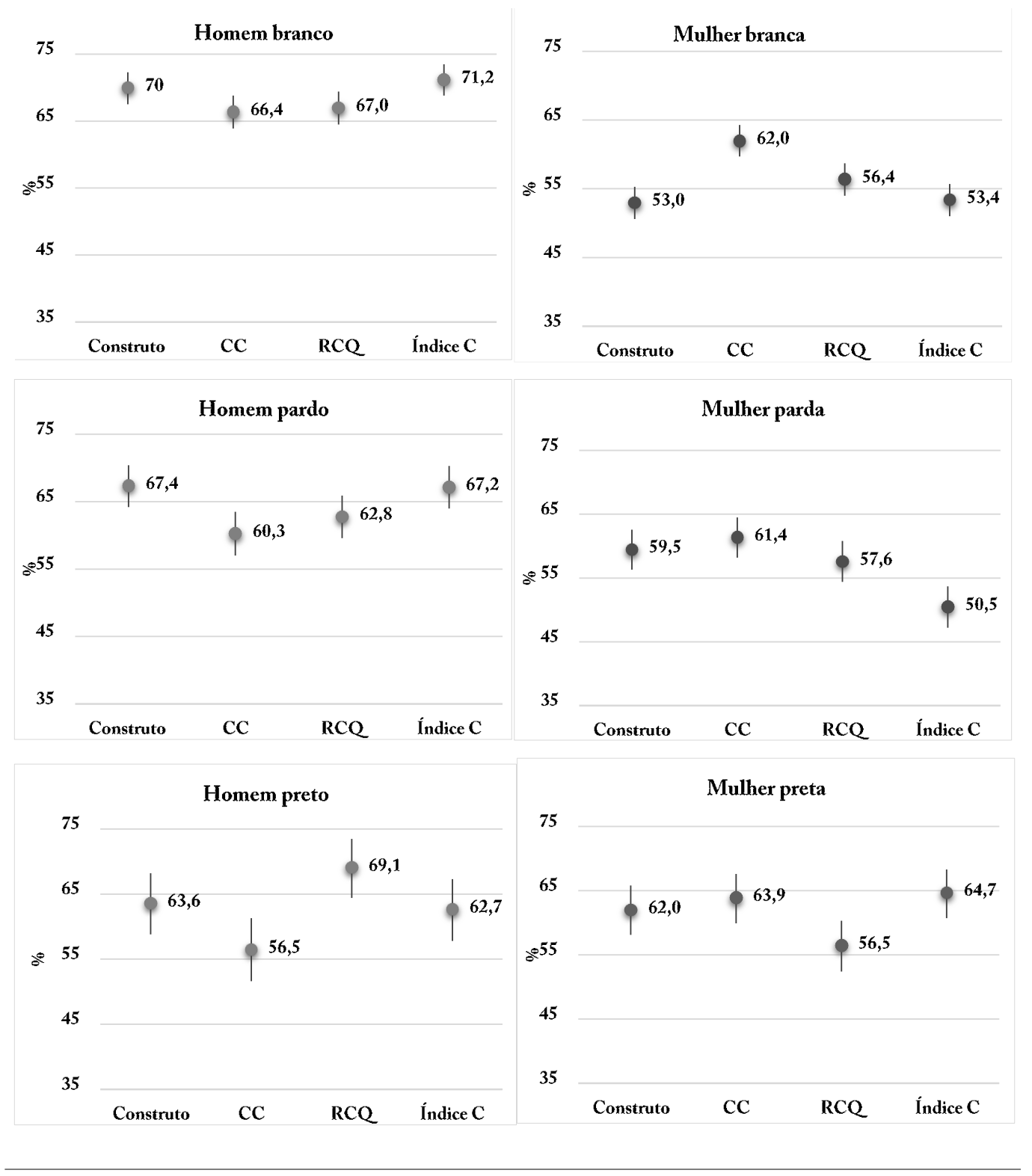

Figura 2. Prevalências de obesidade abdominal e intervalos de $95 \%$ de confiança para a variável latente e os demais indicadores de obesidade abdominal, segundo sexo e raça/cor da pele usando os pontos de corte propostos via variável latente como padrão ouro. ELSA-Brasil 2008-2010.

CC: circunferência da cintura; RCQ: razão cintura quadril; Índice C: índice de conicidade.

prevalências entre homens brancos e mulheres pretas. Entretanto, os maiores valores foram observados entre as mulheres. Estudo realizado com base nos dados do International Day for Evaluation of Abdominal Adiposity (IDEA) ${ }^{3} \mathrm{em}$ países da América Latina e Caribe, inclusive o Brasil, mostrou prevalência de obesidade abdominal similar ao atual estudo para homens (70\%), e superior para mulheres $(76 \%)$ usando os pontos de corte propostos pela IDF.

Concordando parcialmente com os achados do presente artigo, Carroll et al. ${ }^{4}$ relatam que é esperado mulheres e homens pretos com menores quantidades de gordura visceral em comparação 
com hispânicos e brancos, ainda que tenham suas concentrações de biomarcadores inflamatórios similares ou maiores. Por outro lado, homens e mulheres de raça/cor da pele preta possuem maior quantidade de gordura subcutânea que brancos ${ }^{5}$. Participantes do ELSA-Brasil de raça/ cor da pele preta apresentaram maior massa corporal e circunferência do quadril comparados aos brancos e pardos, possivelmente pela maior quantidade de gordura subcutânea. $\mathrm{O}$ acúmulo de gordura subcutâneo, abdominal ou não, também atua como fator de risco à saúde, variando conforme a quantidade e capacidade metabólica do tecido, e pode estar presente independentemente do excesso de gordura visceral ${ }^{34}$. Destacase que, apesar de não ter sido avaliada a gordura visceral neste artigo, as medidas incluídas são indicadores indiretos deste tecido.

Neste estudo, homens e mulheres pardos e pretos apresentaram maior frequência de atividade física classificada como fraca, menor proporção de alta escolaridade e elevada prevalência de obesidade abdominal. A escolaridade pode determinar o estilo de vida adotado pelos sujeitos, abrangendo o padrão alimentar e a prática de atividade física. Além disso, a qualidade do autocuidado e a capacidade de interpretar informações relativas a comportamentos preventivos para a proteção da saúde, inclusive sobre o acúmulo de gordura abdominal, também pode ser influenciada por essa variável ${ }^{21}$.

Quando comparados aos atuais pontos de corte, aqueles sugeridos pela literatura parecem superestimar a obesidade abdominal para a CC em todos os grupos, exceto homens brancos. Pelo índice $\mathrm{C}$ houve subestimação da prevalência entre os homens e superestimação entre as mulheres. O contrário ocorreu com a RCQ. As diferenças nas prevalências chegaram a $15,2 \%$ entre as mulheres brancas e $11 \%$ nos homens pardos para a RCQ. Esses resultados reforçam que os pontos de corte recomendados internacionalmente $\mathrm{e}$ usados no Brasil, de modo geral, não são apropriados, como apontado por outros estudos 9 .

Ainda que esses resultados reflitam as diferenças na natureza dos indicadores de obesidade abdominal e a diversidade biológica segundo raça/ cor da pele e sexo, as prevalências de obesidade abdominal interferem no desenvolvimento de ações de prevenção, promoção e tratamento em saúde, sendo fundamental o uso de pontos de corte acurados para gerar diagnósticos confiáveis em diferentes grupos. Notou-se que resultados referentes à raça/cor da pele parda variaram em torno dos resultados apresentados por pretos e brancos. As disparidades de raça/cor da pele em saúde derivam de complexas relações entre características biológicas, socioeconômicas, ambientais e comportamentais, e variam geograficamente influenciando o padrão de distribuição de gordura corporal ${ }^{1}$. O perfil de distribuição de gordura corporal de pardos ainda é pouco conhecido, uma vez que poucos estudos separam pretos e pardos, dificultando comparações e o estabelecimento de um padrão para a categoria "pardos".

Este foi o primeiro artigo a usar a análise de classes latentes para definir um construto de obesidade abdominal e propor pontos de corte para diferentes indicadores com base nesse padrão -ouro. A validação cruzada, abordagem estatística usada neste estudo para estender os resultados do modelo empírico em outro conjunto de da$\operatorname{dos}^{26}$, permitiu a aplicação do padrão de respostas da variável latente obesidade abdominal em subamostra diferente da qual foi definida inicialmente. Desta forma torna-se possível definir uma variável padrão-ouro de obesidade abdominal em qualquer outra subamostra e garantir a reprodutibilidade do estudo.

A literatura é controversa a respeito dos valores de referência para os indicadores de obesidade abdominal sob investigação. Considerando as diferentes estratégias de investigação entre os estudos, incluindo a abordagem analítica, a comparação com a literatura é limitada. Diferente desta, grande parte das pesquisas não estratificou a amostra por raça/cor da pele e sugeriu pontos de corte para detectar diferentes elementos (risco coronariano elevado, fatores de risco cardiovascular, doença cardiovascular e coronariana, e gordura visceral identificada pela tomografia computadorizada), divergindo do atual estudo que usou uma variável latente como padrão-ouro.

O ELSA-Brasil foi realizado em três grandes regiões geográficas, nas quais residem a maior parte da população brasileira. Isso permite trazer à luz novas informações em saúde considerando a diversidade étnica brasileira, especialmente sobre a população negra. O estudo incluiu adultos e idosos jovens na linha de base. Ainda que se tenha analisado as diferenças entre raça/cor, os dados não foram avaliados por idade, uma vez que para o atual artigo apenas $17 \%$ da amostra tem entre 60 e 74 anos na linha de base. É conhecido que idosos têm mais gordura abdominal que adultos e sugere-se que outros estudos abordem também essa questão. O ELSA-Brasil, por ser uma coorte de trabalhadores não incluiu participantes de camadas mais pobres da população, sugerindo cautela no uso dos achados. Precaução também 
deve ser tomada na aplicação dos atuais pontos de corte em populações com idades diferentes da faixa etária aqui estudada.

É necessário reiterar a imperfeição dos indicadores que definiram o construto de obesidade abdominal pois não estão livres de erros de classificação ${ }^{13}$, embora métodos de imagem para quantificar a gordura abdominal também não sejam isentos desses erros ${ }^{35}$. Para a análise de classes latentes foi necessário classificar previamente os participantes com e sem obesidade abdominal. Apesar desta análise permitir a combinação das informações diagnósticas iniciais, o uso de pontos de corte descritos na literatura nacional e internacional para essa análise pode ter influenciado os resultados. Apesar disso, a metodologia utilizada não distorce a prevalência de obesidade abdominal encontrada pelos indicadores separadamente.

\section{Conclusão}

A maioria dos participantes exibiu obesidade abdominal em todos os subgrupos populacionais avaliados. A CC entre os homens e a RCQ e índice $\mathrm{C}$ entre homens e mulheres apresentaram alto poder para discriminar obesidade abdominal latente, sendo o índice $\mathrm{C}$ o melhor indicador neste estudo. A CC entre as mulheres revelou capacidade moderada em distinguir obesidade abdominal latente. Após aplicar os padrões de resposta obtidos na análise de classes latentes, identificou-se pontos de corte para brancos, pardos e pretos.

Os resultados enfatizam a importância de monitorar a prevalência de obesidade abdominal, dado os altos valores observados em todos os grupos e visam contribuir com um mais adequado diagnóstico de obesidade abdominal individual e coletiva entre os brasileiros. Novos estudos podem ser conduzidos para investigar como esses pontos de corte, segundo sexo e raça/ cor da pele, se relacionam com doenças metabólicas e cardiovasculares. Outrossim, são também possíveis estudos que comparem os indicadores mencionados e a variável latente criada com um método padrão-ouro para avaliação da gordura abdominal.

\section{Colaboradores}

M Eickemberg participou da concepção e delineamento, análise e interpretação dos dados, redação do artigo e aprovação final da versão a ser publicada. LDAF Amorim e SMA Matos participaram da concepção e delineamento, análise e interpretação dos dados, revisão crítica e aprovação da versão final a ser publicada. MCC Almeida, FJG Pitanga, EML Aquino e MJM Fonseca participaram da revisão crítica e aprovação da versão final a ser publicada.

\section{Agradecimentos}

Ao CNPq, FINEP e DECIT pelo financiamento. À toda a equipe do Estudo Longitudinal de Saúde do Adulto (ELSA-Brasil).

O ELSA-Brasil foi financiado pelo Ministério da Saúde (Decit - Departamento de Ciência e Tecnologia) e pelo Ministério da Ciência e Tecnologia do Brasil (Finep -Financiadora de Estudos e Projetos e CNPq - Conselho Nacional de Desenvolvimento Científico e Tecnológico). 


\section{Referências}

1. Balkau B, Deanfield JE, Després JP, Bassand JP, Fox KAA, Smith Junior SC, Barter P, Tan CE, Van Gaal L, Wittchen HU, Massien C, Haffner SM. International day for the evaluation of abdominal obesity (IDEA):A study of waist circumference, cardiovascular disease, and diabetes mellitus in 168000 primary care patients in 63 countries. Circulation 2007; 116(17):1942-1951.

2. Ford ES, Maynard LM, Li C. Trends in mean waist circumference and abdominal obesity among US adults, 1999-2012. JAMA 2014; 312(11):1151-1153.

3. Aschner P, Ruiz A, Balkau B, Massien C, Haffner SM. Association of abdominal adiposity with diabetes and cardiovascular disease in Latin America. J Clin Hypertens 2009; 11(12):769-774.

4. Carroll JF, Fulda KG, Chiapa AL, Rodriquez M, Phelps DR, Cardarelli KM, Vishwanatha JK, Cardarelli R. Impact of race/ethnicity on the relationship between visceral fat and inflammatory biomarkers. Obesity 2009; 17(7):1420-1427.

5. Katzmarzyk PT, Bray GA, Greenway FL, Johnson WD, Newton Junior RL, Ravussin E, Ryan DH, Smith SR, Bouchard C. Racial differences in abdominal depot - specific adiposity in white and African American adults 1 - 3. Am J Clin Nutr 2010; 91(1):7-15.

6. Instituto Brasileiro de Geografia e Estatística (IBGE). Pesquisa nacional de saúde 2013: Ciclos de vida - Brasil e grandes regiões. Rio de Janeiro: IBGE; 2015.

7. Alberti SG, Zimmet P, Shaw J, Grundy SM. The IDF consensus worldwide definition of the metabolic syndrome. Int Diabetes Fed 2006; 1:24.

8. Aschner P, Buendía R, Brajkovich I, Gonzalez A, Figueredo R, Juarez XE, Uriza F, Gomez AM, Ponte CI. Determination of the cutoff point for waist circumference that establishes the presence of abdominal obesity in Latin American men and women. Diabetes Res Clin Pract 2011; 93(2):243-247.

9. Barbosa PJB, Lessa I, Almeida Filho N, Magalhães LBNC, Araújo J. Critério de obesidade central em população brasileira: impacto sobre a síndrome metabólica. Arq Bras Cardiol 2006; 87(4):407-414.

10. Silva DAS, Petroski EL, Peres MA. Accuracy and measures of association of anthropometric indexes of obesity to identify the presence of hypertension in adults: A population-based study in Southern Brazil. Eur J Nutr 2013; 52(1):237-246.

11. Pitanga JFG, Lessa I. Indicadores antropométricos de obesidade como instrumento de triagem para risco coronariano elevado em adultos na cidade de Salvador - Bahia. Arq Bras Cardiol 2005; 85(1):26-31.

12. World Health Organization (WHO). Waist circumference and waist-hip ratio. Report of a WHO expert consultation. Geneva: WHO; 2008.

13. Van Smeden M, Naaktgeboren C, Reitsma JB, Moons KGM, de Groot J A H. Latent class models in diagnostic studies when there is no reference standard--A systematic review. Am J Epidemiol 2014; 179(4):423-431.

14. Fraga DBM, Pacheco LV, Borja LS, Tuy PGSE, Bastos LA, Solcà MS, Amorim LDAFA, Veras PST. The Rapid Test Based on Leishmania infantum Chimeric rK28 Protein Improves the Diagnosis of Canine Visceral Leishmaniasis by Reducing the Detection of False-Positive Dogs. PLoS Negl Trop Dis 2016; 10(1):1-11.
15. O'Neill D. Measuring obesity in the absence of a gold standard. Econ Hum Biol 2015; 17:116-128.

16. Aquino EML, Barreto SM, Bensenor IM, Carvalho MS, Chor D, Duncan BB, Lotufo PA, Mill JG, Molina Mdel C, Mota EL, Passos VM, Schmidt MI, Szklo M. Brazilian Longitudinal Study of Adult health (ELSA -Brasil): Objectives and design. Am J Epidemiol 2012; 175(4):315-324.

17. Schmidt MI, Griep RH, Passos VM, Luft VC, Goulart AC, Menezes GMS, Molina MCB, Alvaro V, Nunes MA. Estrategias e desenvolvimento de garantia e controle de qualidade no ELSA-Brasil. Rev Saude Publica 2013; 47(Supl. 2):105-112.

18. Lohman TG, Roche AF, Martorell R. Anthropometric standardization reference manual. Champaign: Hum Kinet Books; 1988.

19. Centres for Disease Control and Prevention. National health and nutrition examinatory survey (NHANES). Anthropometry procedures manual. Atlanta: NHANES; 2007.

20. Valdez R, Seidell JC, Ahn YI, Weiss KM. A new index of abdominal adiposity as an indicator of risk for cardiovascular disease. A cross-population study. Int $J$ Obes 1993; 17(77):77-82.

21. Ali AT, Crowther NJ. Factors predisposing to obesity: a review of the literature. JEMDSA 2009; 14(2):81-84.

22. Duncan BB, Schimidt MI, Giugliani ERJ. Medicina Ambulatorial: Condutas de Atenção Primária Baseada em Evidências. Porto Alegre: Artmed Editora; 2004.

23. Matsudo S, Araújo T, Matsudo V, Andrade D, Andrade E, Oliveira LC, Braggion G. Questionário internacional de atividade física (Ipaq):estudo de validade e reprodutibilidade no Brasil. Rev Bras Atividade Física Saúde 2001; 6(2):5-18.

24. World Health Organization (WHO). Global strategy on diet, physical activity and health. Geneva: WHO; 2010. 1-60.

25. Mill JG, Pinto K, Griep RH, Goulart A, Foppa M, Lotufo P, Maestri MK, Ribeiro AL, Andreão RV, Dantas EM, Oliveira I, Fuchs SC, Cunha Rde S, Bensenor IM. Medical assessments and measurements in ELSA-Brasil. Rev Saude Publica 2013; 47(2):54-62.

26. Collins LM, Lanza ST. Latent class and latent transition analysis: with applications in the social, behavioral, and health sciences. Hoboken: Wiley; 2010.

27. Yin J, Tian L. Joint confidence region estimation for area under ROC curve and Youden index. Stat Med 2014; 33(6):985-1000.

28. Roriz AKC, Passos LCS, Oliveira CC, Eickemberg M, Moreira PDA, Sampaio LR. Evaluation of the accuracy of anthropometric clinical indicators of visceral fat in adults and elderly. PLoS One 2014; 9(7):1-6.

29. Pitanga FJG, Lessa I. Sensibilidade e especificidade do índice de conicidade como discriminador do risco coronariano de adultos em Salvador, Brasil. Rev Bras Epidemiol 2004; 7(3):259-269.

30. Gustat J, Elkasabany A, Srinivasan S, Berenson GS. Relation of abdominal height to cardiovascular risk factors in young adults: the Bogalusa heart study. $A m$ J Epidemiol 2000; 151(9):885-891. 
31. Zhang X, Shu XO, Gao Y-T, Yang G, Matthews CE, Li Q, Li H, Jin F, Zheng W. Anthropometric predictors of coronary heart disease in Chinese women. Int $\mathrm{J}$ Obes 2004; 28(6):734-740.

32. Carroll JF, Chiapa AL, Rodriquez M, Phelps DR, Cardarelli KM, Vishwanatha JK, Bae S, Cardarelli R. Visceral fat, waist circumference, and BMI: impact of race/ethnicity. Obesity 2008; 16(3):600-607.

33. Carneiro Roriz AK, De Oliveira CC, Moreira PA, Eickemberg M, Barreto Medeiros JM, Sampaio LR. Methods of predicting visceral fat in Brazilian adults and older adults: A comparison between anthropometry and computerized tomography. Arch Latinoam Nutr 2011; 61(1):5-12.

34. Hermsdorff HHM, Monteiro JBR. Gordura visceral, subcutânea ou intramuscular: onde está o problema? Arq Bras Endocrinol Metabol 2004; 48(6):803-811.

35. Campos RMC, Ferreira AP. Validação da absortometria de raio-X de dupla energia para estimativa da gordura corporal relativa de adultos jovens. J Heal Sci Inst 2010; 28(2):145-149.

Artigo apresentado em 27/03/2018

Aprovado em 15/11/2018

Versão final apresentada em 17/11/2018 